\title{
Nicotine Self-Administration Differentially Regulates Hypothalamic Corticotropin-Releasing Factor and Arginine Vasopressin mRNAs and Facilitates Stress-Induced Neuronal Activation
}

\author{
Guoliang Yu, Hao Chen, Wenyuan Zhao, Shannon G. Matta, and Burt M. Sharp \\ Department of Pharmacology, University of Tennessee Health Science Center, Memphis, Tennessee 38163
}

Acute nicotine is a potent stimulus for activation of the stress-responsive hypothalamic-pituitary-adrenal (HPA) axis, while chronic nicotine self-administration (SA) desensitizes the ACTH response to self-administered nicotine but cross-sensitizes to mild footshock stress (mFSS). To identify underlying mechanisms, we investigated (1) the effects of chronic nicotine SA on the coexpression of corticotropin-releasing factor (CRF) and arginine vasopressin (AVP) mRNAs, the primary hypothalamic neuropeptides regulating ACTH release, in the parvocellular division of paraventricular nucleus (pcPVN), and (2) mFSS-induced activation of these neurons during nicotine SA. Adult male Sprague Dawley rats were given $23 \mathrm{~h} / \mathrm{d}$ unlimited access to self-administer nicotine $(0.03 \mathrm{mg} / \mathrm{kg}$ per injection, i.v.) for $19 \mathrm{~d}$. Brains were double labeled with fluorescence in situ hybridization of CRF and AVP mRNAs and triple labeled after mFSS exposure for CRF and AVP mRNAs and c-Fos protein. Chronic nicotine SA significantly increased AVP mRNA signal and the number of pcPVN AVP-positive $\left(\mathrm{AVP}^{+}\right)$neurons (twofold to threefold), reduced the number of CRF-positive $\left(\mathrm{CRF}^{+}\right)$neurons by $\sim 60 \%$, but increased $\mathrm{pcPVN} \mathrm{CRF}{ }^{+} / \mathrm{AVP}^{+}$neuronal number fivefold. Significantly, although chronic nicotine SA did not affect total c-Fos expression induced by $\mathrm{mFSS}$ in pcPVN CRF ${ }^{+}$neurons, the majority of the new $\mathrm{CRF}^{+} / \mathrm{AVP}^{+}$population was activated by this heterotypic stressor. These phenotypic neuronal alterations may provide the pivotal mechanism underlying the capacity of chronically selfadministered nicotine to cross-sensitize the HPA response to specific stressors, suggesting that nicotine may augment HPA responsiveness to specific stressors in human smokers.

Key words: nicotine; self-administration; CRF; AVP; c-Fos; stress

\section{Introduction}

Stress is a well known risk factor for the development and maintenance of smoking (Schepis and Rao, 2005). However, whether smoking itself affects the response to stressors is controversial, because both reductions and increases in stress levels have been reported in smokers (Parrott, 1995; Koval et al., 2004; Croghan et al., 2006; Finkelstein et al., 2006). In animals, acute injection of nicotine, the principal psychoactive component of tobacco smoke, is a potent stimulus for secretion of the stress-responsive hypothalamo-pituitary-adrenal (HPA) axis hormones ACTH (Conte-Devolx et al., 1981; Cam and Bassett, 1983; Matta et al., 1987) and corticosterone (Balfour et al., 1975; Cam and Bassett, 1983). Acutely injected nicotine increased glutamate release and enhanced NMDA receptor activity in noradrenergic regions of the nucleus tractus solitarius projecting to the hypothalamic

Received Aug. 22, 2007; revised Feb. 1, 2008; accepted Feb. 1, 2008.

This work was supported by National Institutes of Health Grant DA-03977 (B.M.S.).

Correspondence should be addressed to Dr. Burt M. Sharp, Department of Pharmacology, University of Tennessee Health Science Center, 874 Union Avenue, Memphis, TN 38163. E-mail: bsharp@utmem.edu.

W.Zhao's present address: Department of Medicine, University of Tennessee Health Science Center, Memphis, TN 38163.

DOI:10.1523/JNEUROSCI.3837-07.2008

Copyright $\odot 2008$ Society for Neuroscience $\quad$ 0270-6474/08/282773-10\$15.00/0 paraventricular nucleus (PVN) (Zhao et al., 2007), projections shown to stimulate norepinephrine release and activate corticotropin-releasing factor (CRF) neurons in PVN (Valentine et al., 1996; Matta et al., 1998). However, the effect of chronically self-administered nicotine on these stress-responsive systems has not been fully resolved.

When chronically self-administered, nicotine appears to function as a stressor, e.g., 14-25 d unlimited access nicotine self-administration (SA) persistently stimulated norepinephrine release, in both PVN (Fu et al., 2001) and amygdala (Fu et al., 2003). We reported that nicotine activated the HPA axis (i.e., increased ACTH and corticosterone levels) on SA day 1, but desensitization occurred by day 3 . However, $20 \mathrm{~d}$ chronic SA sensitized HPA responses to a specific novel stressor, mild footshock stress (mFSS) (Chen et al., 2007a). These findings support previous reports demonstrating HPA desensitization to repeated homotypic stressor exposure, yet hyper-responsiveness to subsequent challenge by novel stressors (i.e., heterotypic stressors) (Aguilera, 1994).

CRF and arginine vasopressin (AVP), both synthesized in PVN, are the primary hypothalamic neuropeptides regulating ACTH secretion during stress (Harbuz and Lightman, 1992). Under specific conditions, CRF and AVP are cosynthesized in 
parvocellular PVN (pcPVN) neurons and cosecreted from median eminence terminals. Chronic exposure to homotypic stressors typically increased pcPVN AVP mRNA levels, whereas CRF mRNA showed varying responses depending on the stress paradigm (Aguilera and Rabadan-Diehl, 2000). Furthermore, repeated stress increased CRF neuronal numbers and terminals coexpressing AVP (de Goeij et al., 1991, 1992; Bartanusz et al., 1993), which may increase the AVP/CRF ratio in pituitary portal circulation during response to a heterotypic stressor. Thus, through interaction with CRF and potentiation of ACTH secretion, AVP may play a critical role in regulating HPA responses to chronic stressors (Aguilera and Rabadan-Diehl, 2000). The potential role of chronic nicotine SA in altering CRF and AVP coexpression within pcPVN neurons remains to be delineated.

Based on evidence that chronically self-administered nicotine functions as a stressor, we hypothesized that chronic nicotine SA would affect CRF/AVP mRNA coexpression in pcPVN. Based on our finding that chronic nicotine SA enhanced ACTH response to a heterotypic stressor (Chen et al., 2007a), we also postulated that chronic nicotine SA would augment activation of pcPVN neurons coexpressing CRF and AVP in response to a novel stressor.

\section{Materials and Methods}

Nicotine self-administration. Adult male Sprague Dawley rats (300-350 g; Harlan, Madison, WI) were given $7 \mathrm{~d}$ to recover from shipping and acclimated to a reversed $12 \mathrm{~h}$ light/dark cycle (lights off at 10:00 A.M.). Standard rat chow and water were provided ad libitum throughout the experiment (except for pair-fed rats; see below). Nicotine (pH 7.2; calculated as free base) was freshly prepared weekly in $200 \mathrm{U}$ of heparinized saline to deliver $0.03 \mathrm{mg} / \mathrm{kg}$ body weight per injection intravenously. SA was performed according to our previously published protocol (Valentine et al., 1997). Briefly, rats received jugular cannulas under xylazineketamine anesthesia (13 and $87 \mathrm{mg} / \mathrm{kg}$ body weight, i.m., respectively; Sigma, St. Louis, MO) and were then individually housed in operant chambers (Coulbourn Instruments, Allentown, PA) inside a soundattenuating, well ventilated environmental chamber. The operant chamber contained two horizontal levers positioned $6 \mathrm{~cm}$ above the floor, and a green cue light $1 \mathrm{~cm}$ above each lever was illuminated when nicotine was available. Lever presses were recorded and syringe pumps controlled by computers and interfaces, using L2T2 or Graphic State software (Coulbourn Instruments). Rats were given $3 \mathrm{~d}$ to recover from jugular surgery, during which time they received antibiotic daily (Baytril, 7.6 $\mathrm{mg} / \mathrm{kg}$ body weight in $0.1 \mathrm{ml}$, i.v.) and hourly computer-driven aliquots $(50 \mu \mathrm{l})$ of $200 \mathrm{U}$ of heparin in saline. After recovery, rats were randomly assigned to treatment groups. Group 1 (free-fed saline SA) received saline intravenously (200 U pf heparin in saline) with each lever press and had ad libitum access to food; group 2 (nicotine SA) received $0.03 \mathrm{mg} / \mathrm{kg}$ body weight nicotine intravenously with each lever press and had ad libitum food access; group 3 (pair-fed saline SA) received saline intravenously (200 U of heparin in saline) with each lever press and received a measured amount of daily food that was equivalent to the amount of food consumed by a matched nicotine SA rat the previous day.

One lever in the operant chamber was randomly designated as the active lever. On SA days, pressing the active lever elicited a computerdriven intravenous injection of nicotine $(0.03 \mathrm{mg} / \mathrm{kg}$ plus $200 \mathrm{U}$ of heparin in $50 \mu \mathrm{l}$ of saline) or saline ( $200 \mathrm{U}$ of heparin in saline) delivered over $0.81 \mathrm{~s}$ on a fix ratio 1 schedule. To prevent excessive dosing, each injection was followed by a $7 \mathrm{~s}$ period during which the green cue light was extinguished and lever presses were recorded, but nicotine was not injected. Pressing the alternate (inactive) lever had no programmed consequence. Rats were given access to nicotine or saline SA $23 \mathrm{~h} / \mathrm{d}$ for $19 \mathrm{~d}$ and learned to self-administer nicotine without previous training, priming, or food deprivation.

The final hour of the lights-on cycle (9:00-10:00 A.M.) was reserved for housekeeping tasks, i.e., refreshing the nicotine solution, performing animal husbandry, resetting the computer program, and downloading data. During this interval, environmental enclosure doors were opened and green cue lights were turned off; levers were not retracted, and lever press activity was not recorded or rewarded during this period. The patency of each jugular line was checked every $2-3 \mathrm{~d}$, and rats with closed lines were excluded from data analysis. All procedures conformed to National Institutes of Health guidelines and were approved by the Institutional Animal Care and Use Committee at the University of Tennessee Health Science Center. In experiments 1 and 3 (i.e., the unstressed cohorts), rats were allowed to self-administer nicotine for $19 \mathrm{~d}$. Because rats reduce their nicotine intake during their inactive phase of the light cycle, rats were killed $4 \mathrm{~h}$ after initiation of the SA day 20 session to ensure sufficient blood nicotine levels, and brains were prepared for dual labeling of CRF and AVP fluorescence in situ hybridization (FISH) labeling or triple labeling of CRF/AVP FISH and c-Fos fluorescence immunocytochemistry.

Blood osmolarity measurement. Blood samples were collected from trunk blood at the time of death after chronic SA and centrifuged at 300 $\mathrm{rpm}$. Serum osmolarity was analyzed according to the instructions of the manufacturer (OSMETTE A; Precision Systems, Natick, MA).

Extinction of nicotine $S A$. In experiment 2 , a separate group of rats $(n=$ 4 nicotine SA and $n=4$ free-fed saline SA) was allowed $19 \mathrm{~d}$ of SA training, as described above. Extinction training (e.g., cue lights inactivated and saline administered instead of nicotine) was initiated on day 20 of nicotine SA; lever presses on both levers were recorded but produced no programmed consequence during the $13 \mathrm{~d}$ of extinction training. Rats were killed $4 \mathrm{~h}$ after the initiation of the extinction session on day 14 (at a diurnal time comparable with that of the nicotine SA rats above), and brains were prepared for FISH labeling.

Mild footshock stress. In experiment 3, a separate group of rats $(n=6$ nicotine SA and $n=6$ free-fed saline SA) were exposed to mFSS. On SA day 20 , rats were allowed to continue routine nicotine SA; $4 \mathrm{~h}$ after initiation of the SA session, rats received five mild electric shocks $(0.6 \mathrm{~mA}$ for $0.5 \mathrm{~s}$ per shock), randomly delivered over $5 \mathrm{~min}$ through a floor grid (model H13-16; Coulbourn Instruments). During the hour immediately after the brief episode of mFSS, lever press behavior was unchanged (Chen et al., 2007a). Rats were killed $1 \mathrm{~h}$ after the last shock, and brains were prepared for CRF and AVP FISH and c-Fos immunohistochemistry.

Riboprobe preparation for CRF and AVP. The CDNA of CRF or AVP was obtained from rat hypothalamic total RNA by reverse transcriptionPCR using the following primer pairs: CRF, GATTTAGGTGACACTATAGAAaaggggaaaggcaaagaaaa and TAATACGACTCACTATAGGGACcgggacttctgttgaggttc; AVP, GATTTAGGTGACACTATAGAAcgacatggagctgagacagt and TAATACGACTCACTATAGGGACaaaaaccctctcgacactcg. These primers contained the promotors for Sp6 or T7 RNA polymerases. The resulting $359 \mathrm{bp}$ CRF fragment and the $260 \mathrm{bp}$ AVP fragment correspond to nucleotides $40-398$ of rat CRF mRNA (GenBank accession number NM_031019) and 134-393 of the rat AVP mRNA (GenBank accession number NM_016992), respectively. The purified CRF or AVP PCR product was used as template CDNA, and CDNA sequences were validated by sequencing. Both the AVP and CRF cDNA sequences are within regions used previously to generate probes used for in situ hybridization (Potter et al., 1994; Ma et al., 1999). The digoxigenin (DIG) or fluorescein Sp6/T7 RNA labeling kit (Roche Diagnostics, Mannheim, Germany) was used to synthesize probes for CRF or AVP, respectively. T7 and Sp6 polymerases were used to synthesize the antisense and sense probes, respectively. Transcription was performed for $2 \mathrm{~h}$ at $37^{\circ} \mathrm{C}$; after separation from unincorporated nucleotides on ProbeQuant G-50 micro Columns (GE Healthcare, Little Chalfont, UK), riboprobe yield was determined at optical density $260 \mathrm{~nm}$.

CRF and AVP FISH. All brains were removed immediately when the animals were killed and stored at $-80^{\circ} \mathrm{C}$ until processing. Serial coronal cryosections $(25 \mu \mathrm{m})$ of PVN [from bregma -1.6 to $-2.0 \mathrm{~mm}$ (Paxinos and Watson, 1986)] were mounted on Superfrost/Plus Microscope slides (Fisher Scientific, Pittsburgh, PA) and stored at $-80^{\circ} \mathrm{C}$. For each brain, three sections containing the largest $\mathrm{PVN}$ profiles $(\sim 1.8 \mathrm{~mm}$ caudal to bregma) were chosen for hybridization to allow for anatomical matching between rats; these sections were at least $50 \mu \mathrm{m}$ apart from each other to avoid double counting of neurons. Briefly, slides were thawed under 
vacuum, fixed with $4 \%$ paraformaldehyde in diethyl pyrocarbonatetreated 0.1 м PBS, followed by quenching with $3 \%$ peroxide in methanol, digestion by proteinase $\mathrm{K}$, acetylation in $0.25 \%$ acetic anhydride, and dehydration through graded alcohols into chloroform. Each section was then incubated with $50 \mu \mathrm{l}$ of hybridization solution (50\% in deionized formamide; Sigma, St. Louis, MO) containing both CRF and AVP probes, covered with Hybrislip (Research Products, Mt. Prospect, IL), and incubated in humidified chambers at $57^{\circ} \mathrm{C}$ overnight. The next day, sections were treated with RNase to remove nonhybridized probe and washed through graded SSC buffers. Nonspecific binding was blocked in TNB buffer (PerkinElmer, Boston, MA). Hybridized CRF probe was detected by incubating in anti-DIG HRP IgG (1:200; Roche Diagnostics) for $30 \mathrm{~min}$ at room temperature (RT), followed by washing in maleic acid wash buffer. CRF signal was amplified and visualized by using TSA Plus Cyanine 3 (Cy3) (1:200, 3 min; PerkinElmer). The slides were then incubated in 3\% peroxide to quench the anti-DIG HRP residues, followed by anti-fluorescein HRP (1:200, 30 min at RT; PerkinElmer) to detect hybridized AVP probe. AVP signal was amplified and visualized by TSA Plus Cyanine 5 (Cy5) (1:200, 3 min). Finally, slides were coverslipped using Vectashield (Vector Laboratories, Burlingame, CA). Precautions were taken to accommodate trichostatin A (TSA) amplification differences between hybridizations; each in situ hybridization was conducted simultaneously on sections from both nicotine SA and saline SA rats, and then each laser was standardized to the saline sections from that specific hybridization run. For CRF mRNA labeling of saline SA brain sections, the $543 \mathrm{~nm}$ laser beam was adjusted to its maximum signal sensitivity, without reaching saturation; for AVP mRNA labeling of saline SA brain sections, the $633 \mathrm{~nm}$ laser beam detection sensitivity was set at $20 \%$ below its maximum to permit accurate detection of any increase in signal intensity.

Triple labeling of CRF/AVP FISH and c-Fos fluorescence immunohistochemistry. Double CRF-positive/AVP-positive $\left(\mathrm{CRF}^{+} / \mathrm{AVP}^{+}\right) \mathrm{FISH}$ was performed as described above, but, before coverslipping, slides were blocked with $2 \%$ normal goat serum and incubated in rabbit anti-c-Fos IgG (1:15,000; Calbiochem, San Diego, CA), overnight at RT. After rinses in PBS, slides were incubated in Alexa 488-conjugated anti-rabbit IgG (1:200; Invitrogen, Carlsbad, CA) for $1 \mathrm{~h}$ and then coverslipped.

Confocal laser microscopy and image analysis. Anatomically matched coronal sections from brains of each cohort were imaged with a confocal laser scanning microscope (Axiovert 100M; Zeiss, Oberkochen, Germany; excitation wavelength, $543 \mathrm{~nm}$ for cyanine $3,633 \mathrm{~nm}$ for cyanine 5 , and $488 \mathrm{~nm}$ for Alexa 488), and a montage of $40 \times$ images was made of an entire unilateral PVN, using laser and photomultiplier settings that had been standardized to PVN sections of simultaneously hybridized saline SA rat brains. Hybridization with the Cy3-CRF and Cy5-AVP sense probes showed no specific labeling at these settings (supplemental Fig. 2, available at www.jneurosci.org as supplemental material). Confocal microscopic images were exported in grayscale mode to NIH ImageJ $1.37 \mathrm{c}$ (http://rsb.info.nih.gov/ij). Each pixel above a fixed background threshold was identified as the hybridization signal, and each pixel cluster was defined as a particle. The background threshold was determined by the mean gray value of an unlabeled brain region outside PVN within the same tissue section in three sections per animal among one set of sections stained simultaneously. The signal intensity of a particle was defined as the product of its area (square micrometers) and mean optical density. Cell counting was done by a single investigator, blinded to treatment group, who identified individual neurons colocalizing 4',6'-diamidino2 -phenylindole-positive nuclei and fluorescence positivity above background; these marked cells were counted by NIH ImageJ. For each rat, the positive cell number and total signal intensity were calculated from the mean values of a unilateral PVN on all three sections.

The $\mathrm{CRF}^{+}$region of PVN was identified de facto as pcPVN (Rho and Swanson, 1989); magnocellular PVN ( $\mathrm{mcPVN}$ ) was delineated by outlining the boundary of the $\mathrm{CRF}^{+}$and $\mathrm{AVP}^{+}$regions on the section, using the third ventricle to standardize the dorsoventral position of the PVN and then subtracting the $\mathrm{CRF}^{+}$area from the $\mathrm{AVP}^{+}$region. $\mathrm{pcPVN}$ subregions were identified as the dorsal pcPVN, located medial to $\mathrm{mcPVN}$, and the ventral pcPVN (inferior to dorsal pcPVN).

Statistics. Two-way ANOVA with repeated measures was used to com-

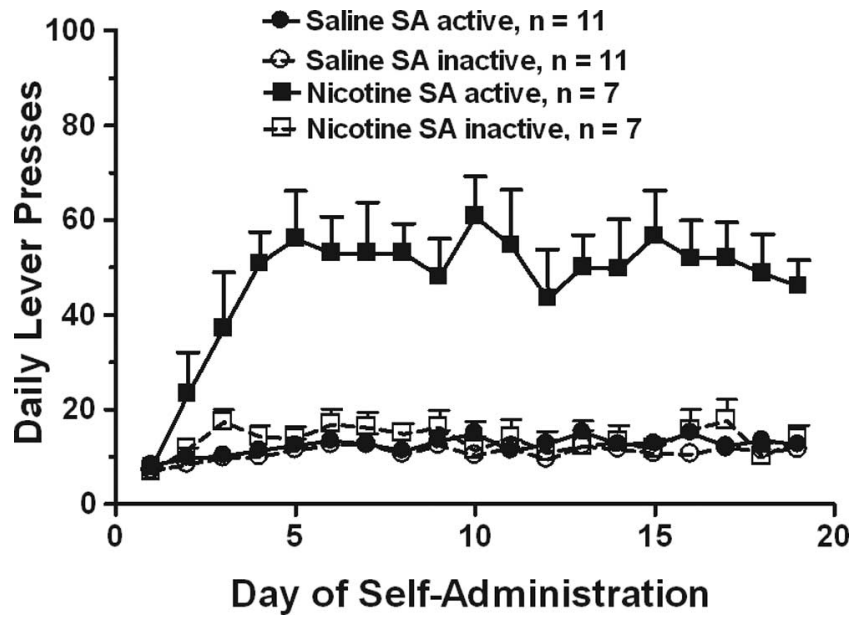

Figure 1. Comparison of daily lever presses between adult male rat cohorts selfadministering nicotine (nicotine $S A ; 30 \mu \mathrm{g} / \mathrm{kg}$ per injection) versus saline. The lever press records from the free-fed and pair-fed saline $S A$ groups were combined, because active $\left(F_{(1,9)}=\right.$ $1.8, p>0.05)$ and inactive $\left(F_{(1,9)}=1.3, p>0.05\right)$ lever presses were similar in both groups. Rats self-administering nicotine pressed significantly more on the active lever than the inactive lever $\left(F_{(1,12)}=28.6, p<0.001\right)$ and also significantly more than the active level presses of control saline $S A\left(F_{(1,16)}=52.1, p<0.001\right)$. There were no significant differences between the active versus inactive lever presses of saline $\operatorname{SA}$ group $\left(F_{(1,20)}=1.3, p>0.05\right)$.

pare the number of lever presses during nicotine and saline SA or extinction, and food intake during nicotine and saline SA, with day treated as a within-subject variable. One-way ANOVA with Scheffé's post hoc comparisons was used to compare differences between treatments in body weight. Student's $t$ test was used to compare the expression of CRF or AVP mRNAs during nicotine and saline SA or extinction. Without direct evidence of a linear relationship between TSA-amplified labeling and mRNA content, all comparisons and fold changes were semiquantitative and throughout the text and figure legends should be considered as approximate fold changes. The effects of SA, mFSS, and anatomical location on the number of $\mathrm{c}-\mathrm{Fos}^{+}$cells were analyzed using factorial ANOVA. In the text and figures, $n$ indicates the number of rats used. Data were presented as mean \pm SEM; statistical significance was assigned at $p<0.05$.

\section{Results}

Lever presses and body weight gain during nicotine SA

Adult male rats acquired nicotine SA $(0.03 \mathrm{mg} / \mathrm{kg}$ body weight per injection, i.v.) without previous training, priming, or food deprivation when the drug was available $23 \mathrm{~h} / \mathrm{d}$, as shown previously (Valentine et al., 1997). In the first experiment, a pair-fed saline SA group was included to control for the potential effects of the reduced food intake induced by nicotine SA on the expression of PVN neuropeptide mRNAs. Figure 1 illustrates the lever press record of all rats that self-administered nicotine versus saline in both experiments 1 and 3 (only unstressed groups); the record of experiment 2, involving nicotine SA and extinction, is presented separately (see Fig. 4). The lever press records from the free-fed and pair-fed groups of experiment 1 were combined, because active $\left(F_{(1,9)}=1.8, p>0.05\right)$ and inactive $\left(F_{(1,9)}=1.3, p>0.05\right)$ lever presses were similar in both groups. Therefore, in Figure 1, the saline SA group represents the combined results of the freefed $(n=4)$ and pair-fed $(n=4)$ saline SA groups from experiment 1 and the saline SA group from experiment 3. In the nicotine SA group (Fig. 1), the number of daily active lever presses was approximately threefold higher than inactive presses $\left(F_{(1,12)}=\right.$ 28.6, $p<0.001)$, whereas active and inactive lever presses were not different in the saline SA group $\left(F_{(1,20)}=1.3, p>0.05\right)$. Additionally, active lever presses were greater in nicotine SA 

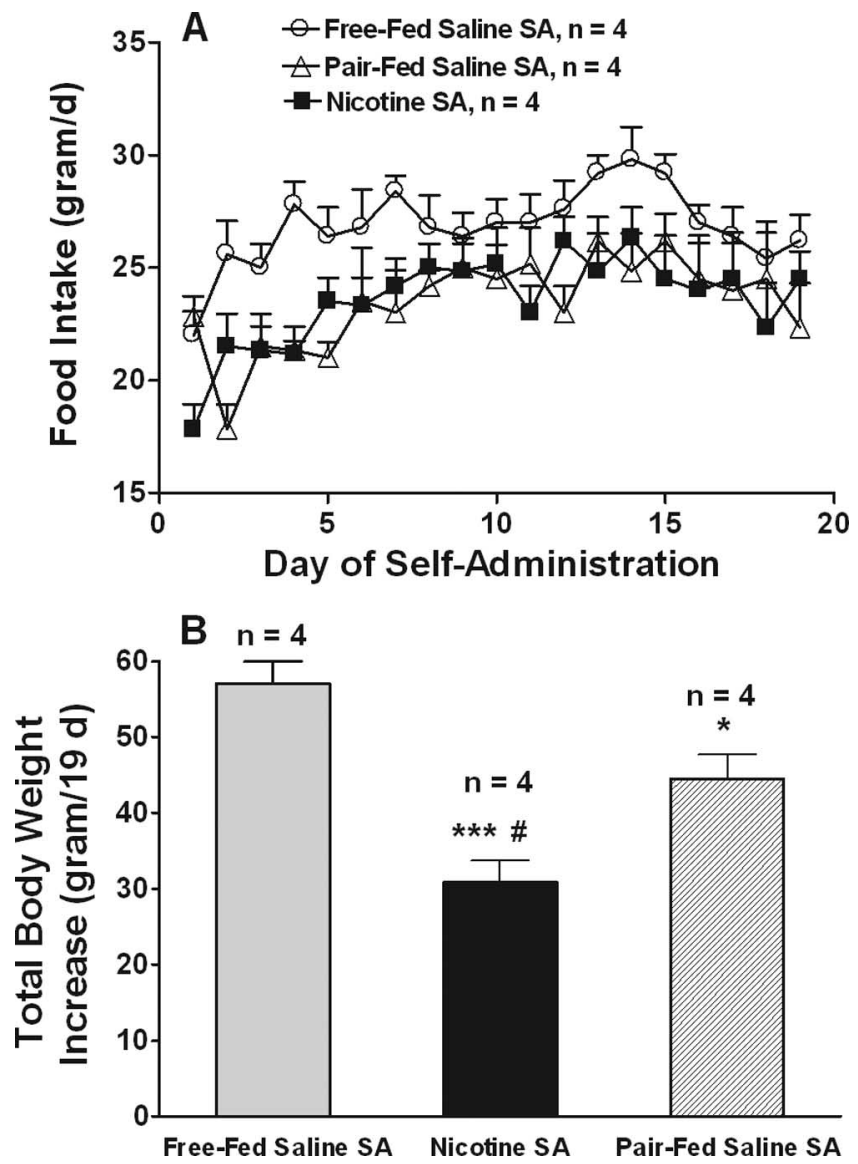

Figure 2. Food intake $(\boldsymbol{A})$ and body weight gain $(\boldsymbol{B})$ during chronic nicotine $S A$. $\boldsymbol{A}$, There was a significant effect of treatment on food intake, in that nicotine $S A$ rats (and their matched pair-fed saline $S A$ controls) ingested less food than free-fed saline $S A$ rats $\left(F_{(1,6)}=8.4\right.$ and 17.7, respectively, $p<0.05)$. $\boldsymbol{B}$, The average body weight increase during $19 \mathrm{~d}$ SA also was significantly different. Nicotine SA rats gained less weight than free-fed saline SA controls $\left({ }^{* * *} p<\right.$ 0.001 ), but they also gained less weight than their pair-fed saline $S A$ controls ( ${ }^{\#} p<0.05$ ), although food intake was matched. ${ }^{*} p<0.05$ compared with free-fed saline $S A$.

compared with saline $\mathrm{SA}\left(F_{(1,16)}=52.1, p<0.001\right)$, but there was no difference in the inactive lever presses $\left(F_{(1,16)}=1.8, p>0.05\right)$.

In experiment 1 , nicotine $S A$ rats ingested less food than freefed saline SA rats $\left(F_{(1,6)}=8.4, p<0.05\right)$, and, as a consequence, the pair-fed saline SA rats also had less food intake than free-fed saline SA rats $\left(F_{(1,6)}=17.7, p<0.05\right)$ (Fig. $\left.2 A\right)$. Although nicotine SA rats gained less weight than free-fed saline SA rats $(p<$ 0.001 ), this reduction could not be attributed solely to reduced food intake because there was a significant difference in body weight gain between the nicotine SA rats and their pair-fed controls $(p<0.05)$ (Fig. $2 B$ ). Indeed, this difference in body weight increase between nicotine SA and pair-fed saline SA accounted for $55.4 \%$ ( $14.5 \pm 2.8$ vs $26.2 \pm 5.6 \mathrm{~g})$ of the difference in weight increase between the nicotine SA and the free-fed saline SA.

\section{CRF and AVP mRNA expression during nicotine SA}

Photomicrographic images and data analyses of CRF and AVP antisense probes FISH are presented in Figures 3 and 4, respectively. In control saline SA rats, the CRF-Cy3 (green)-labeled pcPVN neurons, primarily within the dorsal pcPVN, displayed medium to high fluorescence intensity (Fig. 3A,C), whereas the AVP-Cy5 (red) medium to high intensity labeling was primarily of neurons within mcPVN (Fig. $3 A$ ); only a few pcPVN neurons were $\mathrm{AVP}^{+}$(Fig. $3 A, D$ ), and these did not colocalize the CRF signal (Fig. $3 E$ ). In contrast, in nicotine SA rats, the $\mathrm{AVP}^{+}$cell numbers and signal intensities were increased in both pcPVN and $\operatorname{mcPVN}$ (Fig. $3 B, G$ ), whereas the total number of $\mathrm{CRF}^{+}$neurons and their signal intensities both were markedly decreased (Fig. $3 B, F$ ). However, the number of pcPVN neurons coexpressing CRF and AVP was increased with chronic nicotine SA (Fig. $3 B, H$ ).

Semiquantification of CRF and AVP mRNA expression showed no significant differences between free-fed saline and pair-fed saline SA in the total signal intensity of CRF $(3.6 \pm 0.4 \times$ $10^{5}$ vs $3.8 \pm 0.4 \times 10^{5}$ units, $\left.p>0.05\right)$ or of AVP in either pcPVN $\left(0.7 \pm 0.1 \times 10^{5}\right.$ vs $0.8 \pm 0.1 \times 10^{5}$ unit, $\left.p>0.05\right)$ or $\mathrm{mcPVN}$ $\left(2.0 \pm 0.3 \times 10^{5}\right.$ vs $2.5 \pm 0.2 \times 10^{5}$ units, $\left.p>0.05\right)$. Similarly, there were no significant differences between free-fed saline and pair-fed saline SA in the number of $\mathrm{CRF}^{+}$neurons $(142.8 \pm 6.8$ vs $155 \pm 8.6, p>0.05)$ or of $\mathrm{AVP}^{+}$neurons in either pcPVN $(32.7 \pm 4.4$ vs $32.8 \pm 1.7, p>0.05)$ or $\operatorname{mcPVN}(71.3 \pm 3.3$ vs $69.3 \pm 6.0, p>0.05)$. Last, there was no difference in the number of $\mathrm{CRF}^{+} / \mathrm{AVP}^{+}$neurons between these two saline treatment groups $(3.8 \pm 0.3$ vs $4.1 \pm 0.5, p>0.05)$. Therefore, we combined the free-fed saline SA and pair-fed saline SA groups in Figure 4.

Chronic nicotine SA decreased pcPVN CRF total signal intensity (Fig. $4 A$ ) to $52 \%$ of that in saline SA rats $\left(1.9 \pm 0.2\right.$ vs $3.7 \pm 0.3 \times 10^{5}$ units, $p<0.01)$. In contrast, chronic nicotine SA significantly increased AVP mRNA total signal intensity by $322 \%(2.3 \pm 0.3$ vs $0.7 \pm 0.1 \times 10^{5}$ unit, $\left.p<0.001\right)$ in the $\mathrm{pcPVN}$ and by $230 \%$ in the $\operatorname{mcPVN}\left(5.2 \pm 0.4\right.$ vs $2.2 \pm 0.2 \times 10^{5}$ units, $\left.p<0.001\right)$. Figure $4 B$ showed that the total number of pcPVN CRF ${ }^{+}$neurons in nicotine SA rats decreased by $65 \%(97.2 \pm 6.6$ vs $149 \pm 5.6 ; p<0.001)$. Similarly, the number of pcPVN $\mathrm{AVP}^{+}$neurons in nicotine SA group increased by $189 \%(61.8 \pm 4.3$ vs $32.8 \pm 2.2 ; p<0.001)$. Similar comparisons also showed a $168 \%$ increase (118.2 \pm 5.6 vs $70.3 \pm 3.2, p<0.001)$ in $\mathrm{mcPVN} \mathrm{AVP}^{+}$neurons of nicotine SA rats. Most importantly, chronic nicotine SA significantly increased the number of pcPVN $\mathrm{CRF}^{+} / \mathrm{AVP}^{+}$neurons fivefold compared with saline SA rats $(21.3 \pm 3.8$ vs $4.0 \pm 0.3, p<0.001)$; the percentage of $\mathrm{CRF}^{+} / \mathrm{AVP}^{+}$neurons in $\mathrm{CRF}^{+}$neurons was increased from $2.7 \pm$ 0.2 to $22 \pm 4 \%(p<0.001)$ between saline SA and nicotine SA group. Finally, the difference in signal intensity and/or neuronal number related to nicotine SA could not be attributed to druginduced alterations in osmolarity because serum osmolarity in saline SA and nicotine SA rats was comparable (304.8 \pm 0.9 vs $304.7 \pm 0.9$ mOsm, $p>0.05)$.

\section{Lever presses and CRF and AVP mRNA expression during extinction of nicotine SA}

To determine whether nicotine SA is required to sustain the foregoing changes in the expression of PVN CRF and AVP mRNAs, the behavior was extinguished in the second experiment, as shown in Figure 5A. Nicotine SA was extinguished after $19 \mathrm{~d}$ by inactivating the cue lights and eliminating the nicotine reinforcement, while the levers were available without consequence. In general, the number of active lever presses decreased progressively over time during the $13 \mathrm{~d}$, and the active lever presses during the last $3 \mathrm{~d}$ of extinction were significantly less than the last $3 \mathrm{~d}$ of nicotine $\mathrm{SA}\left(F_{(1,6)}=10.5\right.$, $p<0.05)$. In addition, there was no difference between active and inactive lever presses during the last $3 \mathrm{~d}$ of extinction in nicotine SA rats $\left(F_{(1,6)}=1.8, p>0.05\right)$. In saline SA rats, there also was no difference throughout extinction in active and inactive lever presses $\left(F_{(1,6)}=2.3, p>0.05\right)$.

Extinction training (and/or the concurrent withdrawal from nicotine) reversed the CRF and AVP mRNA changes induced by chronic nicotine SA. Specifically, as shown in Figure $5 B$, total 

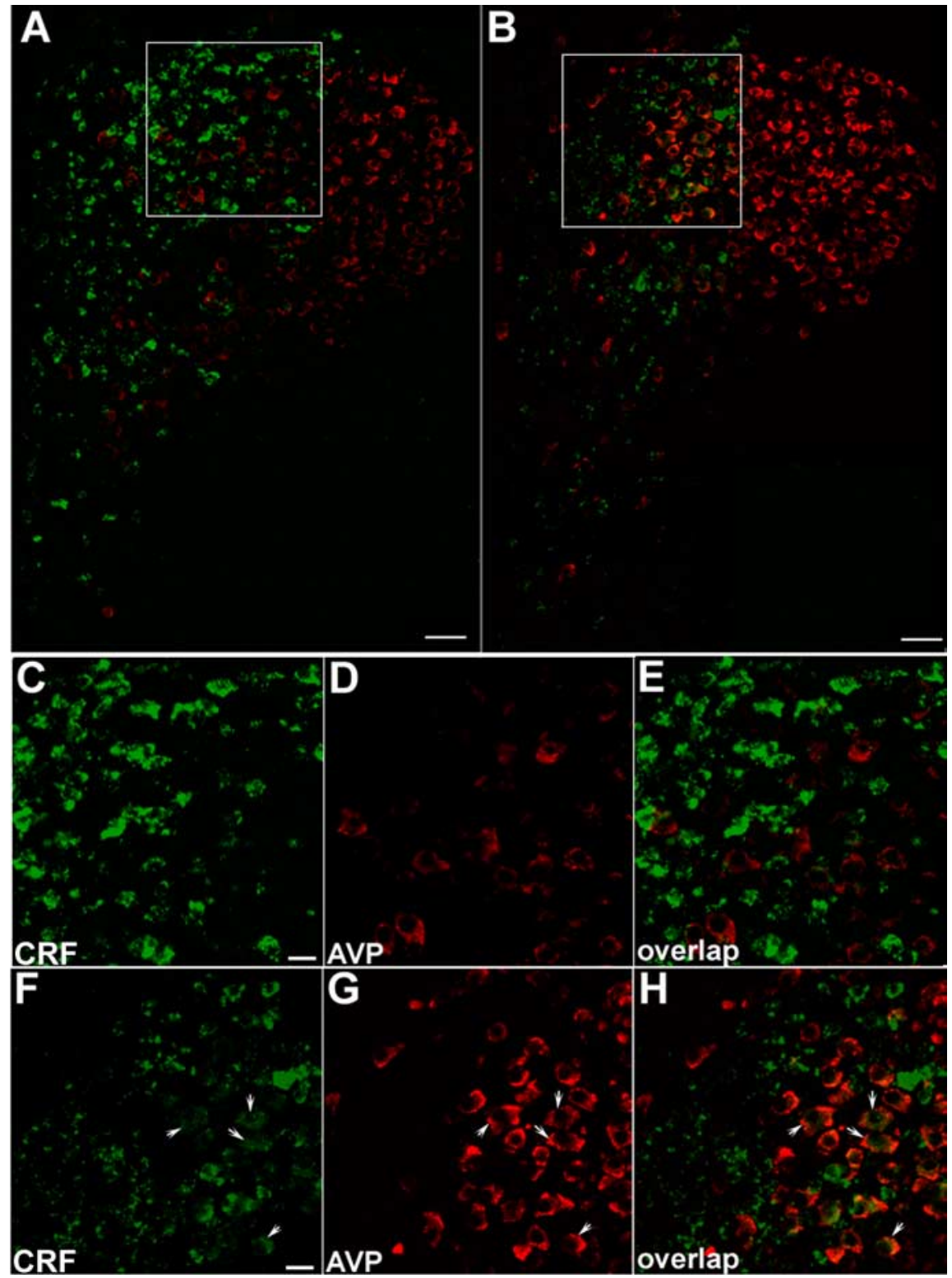

Figure 3. Chronic nicotine SA alters PVN CRF and AVP mRNA expression. Photomicrographs illustrate FISH labeling of Cy3-CRF (green) and Cy5-AVP (red) antisense cRNA probes throughout the PVN of representative saline SA and nicotine SA rats after $19 \mathrm{~d}$ $S A$. Top row show the coronal PVN profiles of $\mathrm{CRF}^{+}$and $\mathrm{AVP}^{+}$neurons in representative saline $\mathrm{SA}(\boldsymbol{A})$ and nicotine $\mathrm{SA}(\boldsymbol{B})$ rats, with the third ventricle (not visible) along the medial (left) border and each outlined box indicating the higher-magnification insets in $\boldsymbol{C}-\boldsymbol{E}$ and $\boldsymbol{F}-\boldsymbol{H}$, respectively. In saline $S A$ rats, a large number of $C R F^{+}$neurons of medium to high fluorescence intensity were visible primarily in the dorsal pcPVN $(A, C)$, whereas AVP ${ }^{+}$neurons were found primarily in the $\mathrm{mCPVN}$ and the few AVP ${ }^{+}$ neurons in pcPVN $(\boldsymbol{A}, \boldsymbol{D})$ showed no $\mathrm{CRF}^{+}{ }^{+}$colocalization $(\boldsymbol{E})$. In contrast, in nicotine SA rats, the $\mathrm{CRF}^{+}{ }^{+}$number and signal intensity were markedly decreased $(\boldsymbol{B}, \boldsymbol{F})$, whereas AVP ${ }^{+}$cell numbers and intensities were increased in both the pcPVN and $\mathrm{mcPVN}(\boldsymbol{B}, \boldsymbol{G})$. In addition, in nicotine $S A$ rats, there was a significant increase in the number of pcPVN neurons colocalizing CRF and AVP mRNAs $(\boldsymbol{H})$ compared with the number of $\mathrm{CRF}^{+} / \mathrm{AVP}^{+}$neurons in saline $S A$ rats $(\boldsymbol{E})$. Arrows indicate representative colocalized neurons in $\boldsymbol{F}-\boldsymbol{H}$. Scale bars: $\boldsymbol{A}, \boldsymbol{B}, 50 \mu \mathrm{m} ; \boldsymbol{C}-\boldsymbol{H}, 20 \mu \mathrm{m}$.

signal intensity was not different between nicotine SA and saline SA rats for pcPVN CRF $\left(4.1 \pm 0.9\right.$ vs $4.0 \pm 0.2 \times 10^{5}$ units, $p>$ $0.05)$ or for AVP in either pcPVN $\left(0.7 \pm 0.2\right.$ vs $0.8 \pm 0.2 \times 10^{5}$ units, $p>0.05)$ or $\operatorname{mcPVN}\left(2.1 \pm 0.3\right.$ vs $2.9 \pm 0.8 \times 10^{5}$ units, $p>0.05$ ). Similarly, the numbers (Fig. $5 C$ ) of pcPVN CRF ${ }^{+}$ $(37.3 \pm 9.5$ vs $141 \pm 2.1, p>0.05)$ and $\mathrm{AVP}^{+}$neurons in pcPVN $(29.8 \pm 3.7$ vs $36.2 \pm 2.1, p>0.05)$ or $\operatorname{mcPVN}(65.6 \pm 3.4$ vs $77.7 \pm 6.8, p>0.05)$ were not different between nicotine and saline SA rats.
Effects of chronic nicotine SA on c-Fos basal expression and induction by $\mathrm{mFSS}$ Nicotine SA has been reported to enhance the HPA response to mFSS (Chen et al., 2007a). Therefore, the third experiment was performed to determine the responsiveness of subsets of phenotypically defined PVN neurons to mFSS. Photomicrographic images and data analyses of mFSS experiments are presented in Figures 6 and 7 , respectively, with a supplemental image demonstrating basal c-Fos expression in the absence of mFSS (supplemental Fig. 1, available at www.jneurosci.org as supplemental material). After $19 \mathrm{~d}$ SA, but without the novel mFSS stressor, few c-Fos ${ }^{+}$ cells (blue) were found in the PVN of saline SA rats, and the low level of c-Fos labeling in nicotine SA rats was colocalized to $\mathrm{CRF}^{+}$(green) or $\mathrm{AVP}^{+}$(red) neurons but rarely observed in neurons colocalizing CRF and AVP (supplemental Fig. 1, available at www.jneurosci.org as supplemental material). mFSS induced c-Fos expression in the PVN of both saline and nicotine SA rats (Fig. $6 A, B$, respectively). In saline $S A$ rats, a moderate number of neurons double labeled for c-Fos and $\mathrm{CRF}^{+}$or $\mathrm{AVP}^{+}$, but few cells were positive for all three (Fig. $6 A, C-F$ ). In nicotine SA rats, although the numbers of $\mathrm{c}-\mathrm{Fos}^{+} /$ $\mathrm{CRF}^{+}$neurons were comparable with those of saline SA rats, there were more $\mathrm{c}-\mathrm{Fos}^{+} / \mathrm{AVP}^{+}$neurons in both pcPVN and mcPVN. Moreover, nearly sixfold more neurons, primarily in dorsal pcPVN, contained all three signals in nicotine SA compared with saline SA rats (Fig. 6B, J).

When analyzed using factorial ANOVA (Fig. 7), c-Fos ${ }^{+} / \mathrm{AVP}^{+}$neuronal number was shown to be affected by SA treatment (nicotine $>$ saline, $F_{(1,28)}=27.9, p<$ 0.001 ), by mFSS (shock $>$ no shock, $F_{(1,28)}$ $=71.5, p<0.001)$, and by PVN division $\left(\operatorname{mcPVN}>\operatorname{pcPVN}, F_{(1,28)}=57.0, p<\right.$ 0.001). Furthermore, there were significant interactions between SA treatment and shock $\left(F_{(1,28)}=21.2, p<0.001\right)$ and PVN division and shock $\left(F_{(1,28)}=13.4\right.$, $p<0.01)$ but not among PVN division, SA treatment and shock $\left(F_{(1,28)}=3.9, p>\right.$ 0.05). Therefore, both mcPVN and pcPVN $\mathrm{AVP}^{+}$neurons responded to $\mathrm{mFSS}$, and chronic nicotine SA significantly enhanced the pcPVN AVP ${ }^{+}$neuronal response to $\mathrm{mFSS}$.

The number of c-Fos ${ }^{+} / \mathrm{CRF}^{+}$neurons (Fig. 7) also was affected by mFSS ( shock $>$ control, $F_{(1,28)}=89.1, p<0.001$ ), by pcPVN division (dorsal $>$ ventral, $F_{(1,28)}=76.2, p<0.001$ ), and by their interaction $\left(F_{(1,28)}=19.5, p<0.001\right)$ but not by SA treatment $\left(F_{(1,28)}=0.5 ; p>0.05\right)$ or its interaction with shock $\left(F_{1,28}=1.0 ; p>0.05\right)$ or $\mathrm{pcPVN}$ division $\left(F_{1,28}=1.7, p>0.05\right)$ or the interaction among pcPVN division, SA treatment, and shock $\left(F_{(1,28)}=3.9, p>0.05\right)$. Therefore, although mFSS in- 

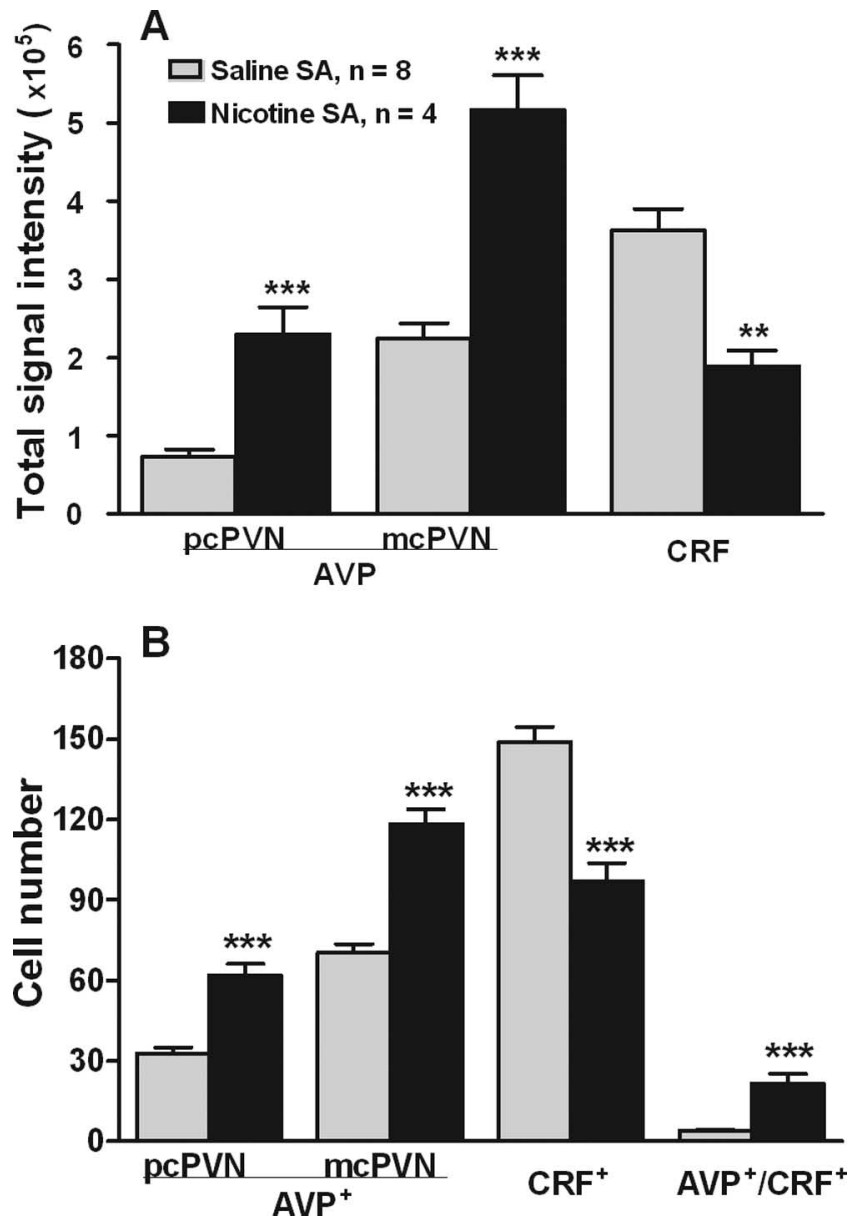

Figure 4. Quantitative analysis of CRF and AVP mRNA labeling after chronic nicotine SA. $\boldsymbol{A}$, The total AVP signal intensities in mcPVN and pcPVN of nicotine SA rats were approximately twofold to threefold higher than in the saline SA cohort [*** $p<0.001$ compared with saline SA (pooled free-fed + pair-fed)]. In contrast, CRF signal intensity in nicotine $S A$ rats was reduced by $\sim 50 \%\left({ }^{* *} p<0.01\right)$. B, Chronic nicotine SA increased the number of AVP ${ }^{+}$neurons nearly twofold in both pcPVN and $\mathrm{mcPVN}\left({ }^{* * *} p<0.001\right)$. Nicotine $S A$ resulted in a $60-70 \%$ reduction in $\mathrm{CRF}^{+}$neuronal number in pcPVN $\left({ }^{* * *} p<0.01\right)$ but elicited a fivefold increase in the number of neurons coexpressing (RF and AVP (*** $p<0.001)$.

duced significant activation of $\mathrm{PVN} \mathrm{CRF}^{+}$neurons, chronic nicotine SA did not further enhance the response in this population. In contrast, however, the number of $\mathrm{c}-\mathrm{Fos}^{+} / \mathrm{CRF}^{+} / \mathrm{AVP}^{+}$neurons was significantly affected by SA treatment (nicotine $>$ saline, $\left.F_{(1,28)}=50.9, p<0.001\right), \mathrm{mFSS}$ (shock $>$ control, $F_{(1,28)}=$ $59.5, p<0.001$ ), and by PVN division (dorsal $>$ ventral pcPVN, $\left.F_{(1,28)}=90.1, p<0.001\right)$. A significant interaction also was found between shock and SA treatment $\left(F_{(1,28)}=33.1, p<0.001\right)$, between SA treatment and PVN division $\left(F_{(1,28)}=37.6, p<\right.$ $0.001)$, between shock and PVN division $\left(F_{(1,28)}=41.5, p<\right.$ $0.001)$, and among pcPVN divisions, SA treatment and shock $\left(F_{(1,28)}=26.2, p<0.001\right)$. The percentage of $\mathrm{c}-\mathrm{Fos}^{+} / \mathrm{CRF}^{+} /$ $\mathrm{AVP}^{+}$neurons compared with c-Fos ${ }^{+} / \mathrm{CRF}^{+}$neurons was significantly increased from $12.5 \pm 3.0$ to $40.5 \pm 3.4 \%(p<0.001)$ between saline SA and nicotine SA group in dorsal pcPVN but not in ventral $\mathrm{pcPVN}(5.3 \pm 1.2$ to $10.3 \pm 2.2 \%, p>0.05)$. Therefore, mFSS induced significant activation of $\mathrm{PVN} \mathrm{CRF}^{+} /$ $\mathrm{AVP}^{+}$neurons, with a stronger response in dorsal compared with ventral pcPVN, and chronic nicotine SA further enhanced this heterotypic stress activation.
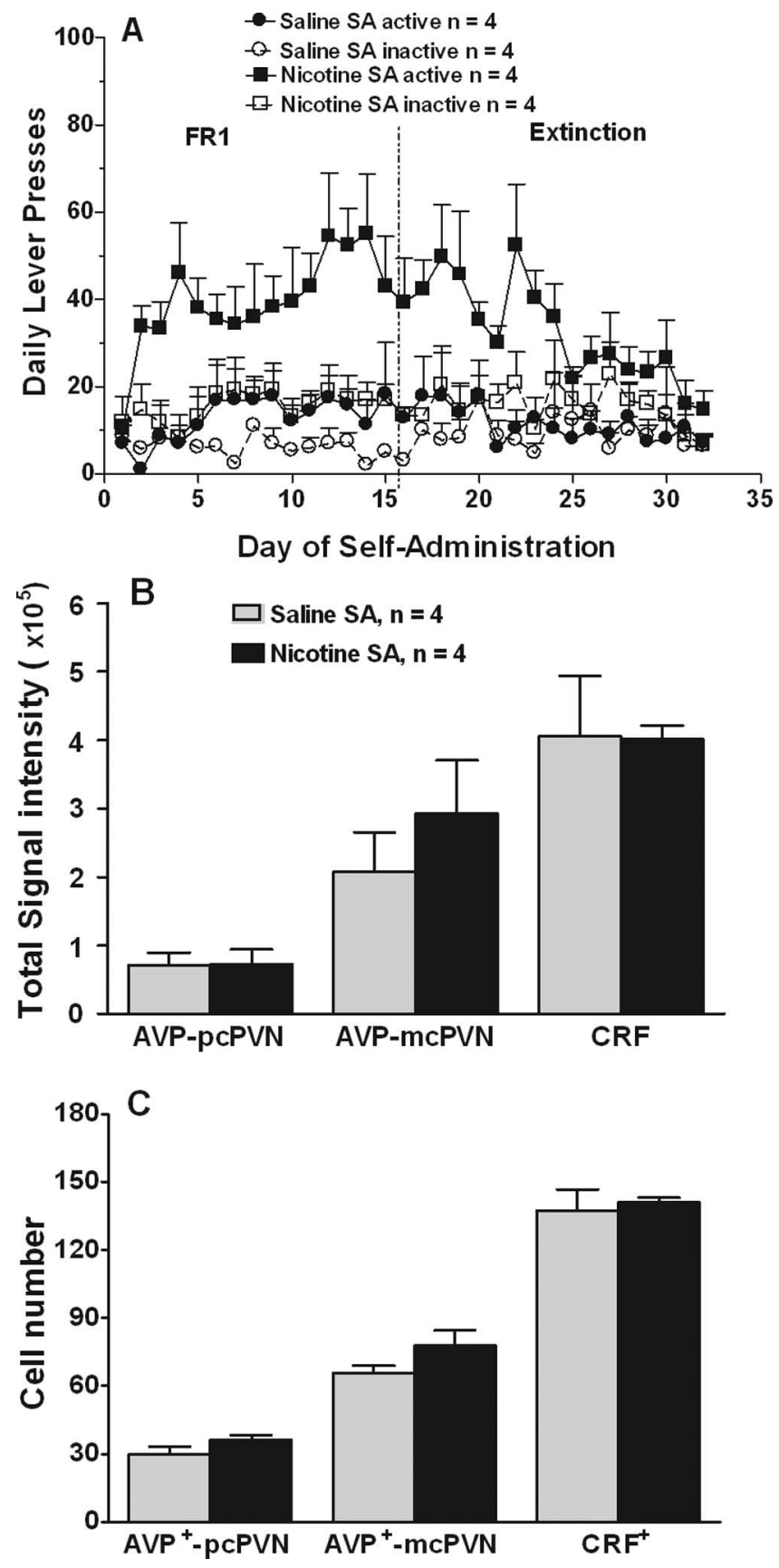

Figure 5. Extinction of chronic nicotine SA: lever press activity and effects on CRF and AVP mRNA expression. $A$, After $S A d 19$ (vertical dashed line), nicotine injections and cue lights were terminated for both the nicotine $S A$ and saline $S A$ cohorts. Active lever presses by the nicotine $S A$ rats declined during the $13 \mathrm{~d}$ of extinction training, so that the average daily active lever presses during last 3 consecutive days of extinction was significantly lower than that during the last 3 consecutive days of nicotine $S A\left(F_{(1,6)}=10.5, p<0.05\right)$ and no different from inactive presses $\left(F_{(1,6)}=1.8, p>0.05\right)$. There were no significant differences between active and inactive lever presses by saline $S A$ rats throughout extinction $\left(F_{(1,6)}=2.3, p>0.05\right)$. FR1, Fixed ratio 1. B, At the end of 13 dextinction training, total signal intensities of (RF, as well as AVP in pcPVN and $\mathrm{mcPVN}$, were no longer different between nicotine SA and either saline SA group ( $p>0.05)$.C, Similarly, after extinction, $\mathrm{CRF}^{+}$cell numbers, as well as $\mathrm{AVP}^{+}$neuronal numbers in pcPVN and $\mathrm{m} C \mathrm{PVN}$, were not different between nicotine and saline SA rats $(p>0.05)$.

\section{Discussion}

With $23 \mathrm{~h}$ ad libitum access to nicotine, chronic (19 d) nicotine SA decreased CRF mRNA expression 50\%, increased AVP mRNA expression twofold to threefold in pcPVN and mcPVN, 


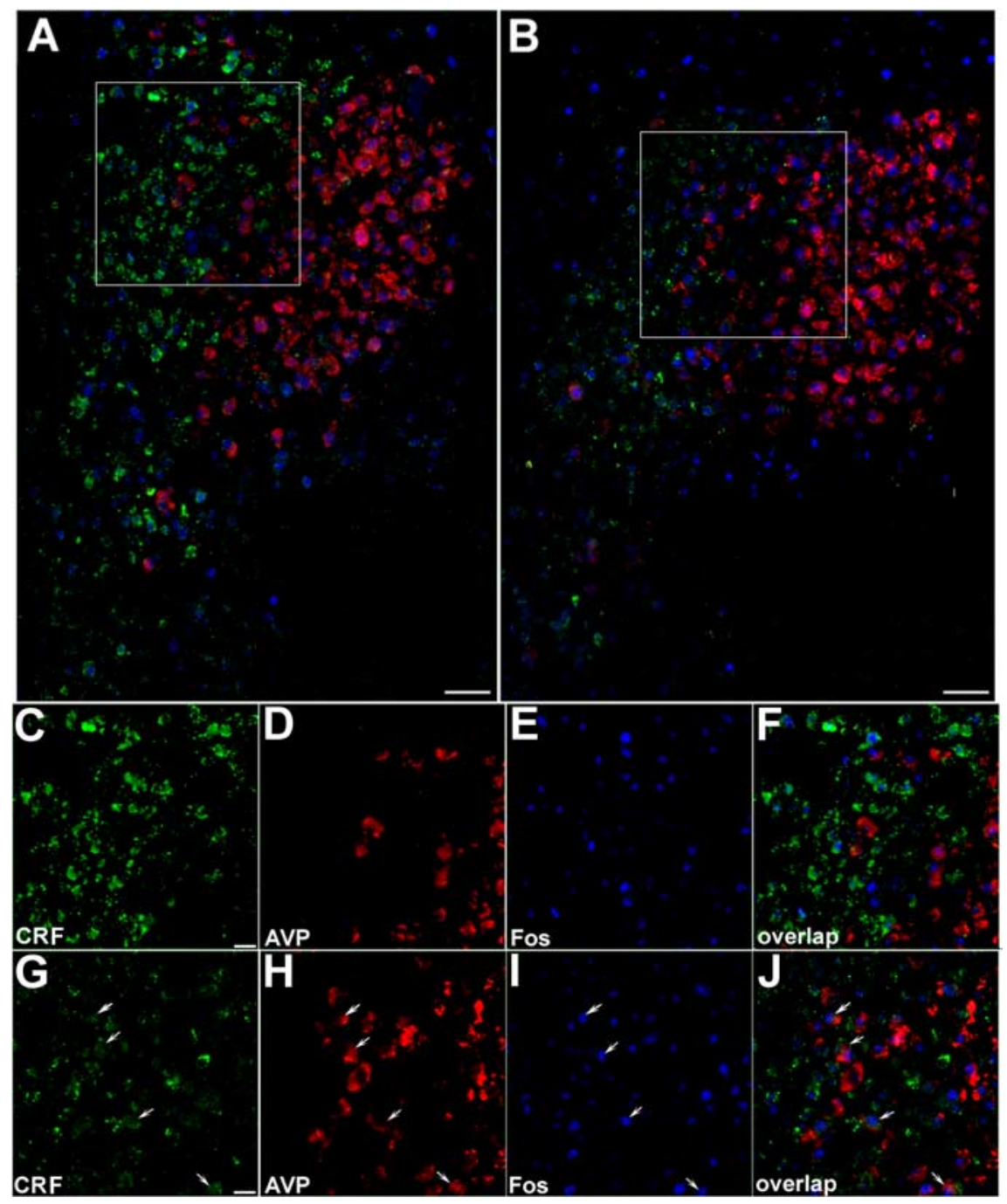

Figure 6. Activation of PVN neurons by a heterotypic stressor (mFSS) during chronic nicotine SA. Top row show the coronal PVN profiles of $\mathrm{CRF}^{+}$(green) and $\mathrm{AVP}^{+}$(red) neurons activated by mFSS and expressing c-Fos protein (blue) in representative saline SA $(\boldsymbol{A})$ and nicotine SA $(\boldsymbol{B})$ rats on SA day 20; each outlined box indicates the higher-magnification insets in $\boldsymbol{C}-\boldsymbol{F}$ and $\mathbf{G}-\boldsymbol{J}$, respectively. In saline SA rats, mFSS induced the expression of c-Fos ${ }^{+}$in the PVN $(\boldsymbol{A}, \boldsymbol{E})$, with a moderate number of $\mathrm{CRF}^{+}$or AVP $^{+}$neurons double labeled for c-Fos, but few cells triple labeled $(\boldsymbol{F})$. In nicotine SA rats, similar to Figure 4, there was a reduction in $\operatorname{CRF}(\boldsymbol{B}, \boldsymbol{G})$ and an increase in $\operatorname{AVP}(\boldsymbol{B}, \boldsymbol{H})$ labeling. Furthermore, many $p c P V N$ neurons were positive for all three labels $(\boldsymbol{J})$, as indicated by the arrowheads. Scale bars: $\boldsymbol{A}, \boldsymbol{B}, 50 \mu \mathrm{m} ; \boldsymbol{C}-\boldsymbol{J}, 20 \mu \mathrm{m}$.

and increased the number of CRF neurons coexpressing AVP mRNA fivefold. Although these changes were reversible by extinction training, they were likely to be responsible for the enhanced stress response after nicotine SA (Chen et al., 2007a), particularly because chronic nicotine SA significantly increased c-Fos activation of $\mathrm{CRF}^{+} / \mathrm{AVP}^{+}$neurons by mFSS. Figure 8 is a schematic of the neuroadaptive changes in the PVN and HPA axis from the acquisition through maintenance of chronic nicotine SA.

Confounding factors contributing to PVN gene expression changes are nicotine-induced reduction in food intake and weight gain (McNair and Bryson, 1983). However, a previous study reported that expression of CRF and AVP mRNA was unaffected by restricting feeding to $2 \mathrm{~h} / \mathrm{d}$ (Johnstone et al., 2005). We also found that limiting saline SA rat food intake, by pair feeding to nicotine SA rats, limited body weight gain (Fig. 2) but did not affect CRF or AVP mRNA expression (Fig. 5). Therefore, reduced food intake was unlikely to contribute to the PVN gene expression changes found in nicotine SA rats. Consistent with a study showing that mice chronically exposed to cigarette smoke had lower body weight than pairfed controls (Chen et al., 2006), nicotine SA rats gained less than pair-fed saline SA rats. Thus, nicotine affects weight gain by both reducing food intake and accelerating energy metabolism and fat utilization (Chen et al., 2007b).

The permanence of the gene expression changes induced by nicotine SA is a crucial issue. A similar decrease in hypothalamic CRF mRNA expression, induced by chronic binge-pattern cocaine administration, was reversible by withdrawal from the drug (Zhou et al., 1996), but a subsequent solution hybridization study did not detect an effect of chronic cocaine or its withdrawal on vasopressin mRNA levels (Zhou et al., 2005). In the current study, after $13 \mathrm{~d}$ nicotine SA extinction training, changes in $\mathrm{CRF}^{+}, \mathrm{AVP}^{+}$, and $\mathrm{CRF}^{+}$/ $\mathrm{AVP}^{+}$neurons were no longer evident, indicating that the adaptation of the HPA axis to chronic nicotine $\mathrm{SA}$ also was reversible.

Decreased CRF expression in pcPVN, as reported here, has been associated with desensitization of ACTH and corticosterone responses to chronic repetition of the same stressor, such as immobilization, restraint, or lipopolysaccharide injection (Hauger et al., 1988; Ma and Lightman, 1998; Grinevich et al., 2001). We reported recently that $\mathrm{ACTH}$ and corticosterone responses to self-administered nicotine completely desensitized within $3 \mathrm{~d}$ (Chen et al., 2007a). Although untested, it is possible that reduced CRF mRNA expression also underlies this desensitization, especially if nicotine SA resulted in the release of only CRF from pcPVN neurons containing both CRF and AVP or if nicotine SA favored activation of neurons containing only CRF. We reported previously that basal ACTH and corticosterone levels were unaffected by chronic nicotine SA (Chen et al., 2007a). Therefore, it is likely that other mechanisms, perhaps compensatory, exist for maintaining basal ACTH and corticosterone levels despite the significant reduction in CRF mRNA levels observed by day 19 of nicotine SA.

Although such compensatory mechanisms are likely to be complex, AVP may be involved. AVP, itself a weak stimulus for ACTH secretion, acts synergistically with CRF, both in vitro (Gillies et al., 1982) and in vivo (Rivier and Vale, 1983), to enhance ACTH secretion from the anterior pituitary. AVP expression in pcPVN generally is increased by repeated homotypic stressors (Aguilera and Rabadan-Diehl, 2000). For example, repeated immobilization increased pcPVN AVP expression and its colocalization with CRF in the median eminence (de Goeij et al., 1991). In addition, the AVP- $1 \mathrm{~b}$ receptor located on anterior pituitary corticotropes is required for normal ACTH response during chronic restraint stress (Lolait et al., 2007). Therefore, the two- 


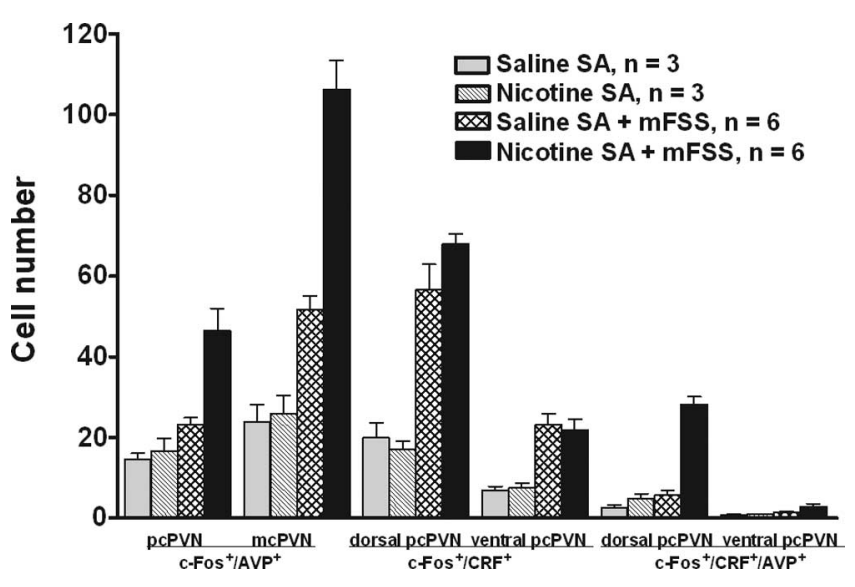

Figure 7. Quantitative analysis of PVN neuronal activation by a heterotypic stressor, $m F S S$, during chronic nicotine SA. In contrast to saline SA rats with or without mFSS exposure or nicotine $S A$ rats without $m F S S$, exposure to the novel stressor in nicotine $S A$ rats increased the number of AVP ${ }^{+}$neurons expressing c-Fos protein $\left(\mathrm{c}-\mathrm{Fos}^{+} / \mathrm{AVP}^{+}\right)$in both $\mathrm{pcPVN}$ and mcPVN $\left(p<0.001\right.$ for each). However, $\mathrm{mFSS}$ elicited comparable $\mathrm{c}-\mathrm{F}$ os induction in $\mathrm{CRF}^{+}$neurons (c-Fos ${ }^{+} / \mathrm{CRF}^{+}$) of both saline SA and nicotine SA rats, regardless of PVN subdivision. Significantly, the neuronal population most affected by mFSS was the recently created $\mathrm{CRF}^{+} / \mathrm{AVP}^{+}$ colocalized neurons induced by chronic nicotine $S A$ in the dorsal pcPVN and that project to the median eminence $\left(c-\mathrm{Fos}^{+} / \mathrm{CRF}^{+} / \mathrm{AVP}^{+} ; p<0.001\right)$.

\section{A: Desensitization of ACTH response to nicotine SA: Day 1: ACTH $\uparrow$ \\ Day 3: ACTH - - NC}

\section{B: Changes in PVN mRNA expression and HPA stress responsiveness during chronic nicotine SA}

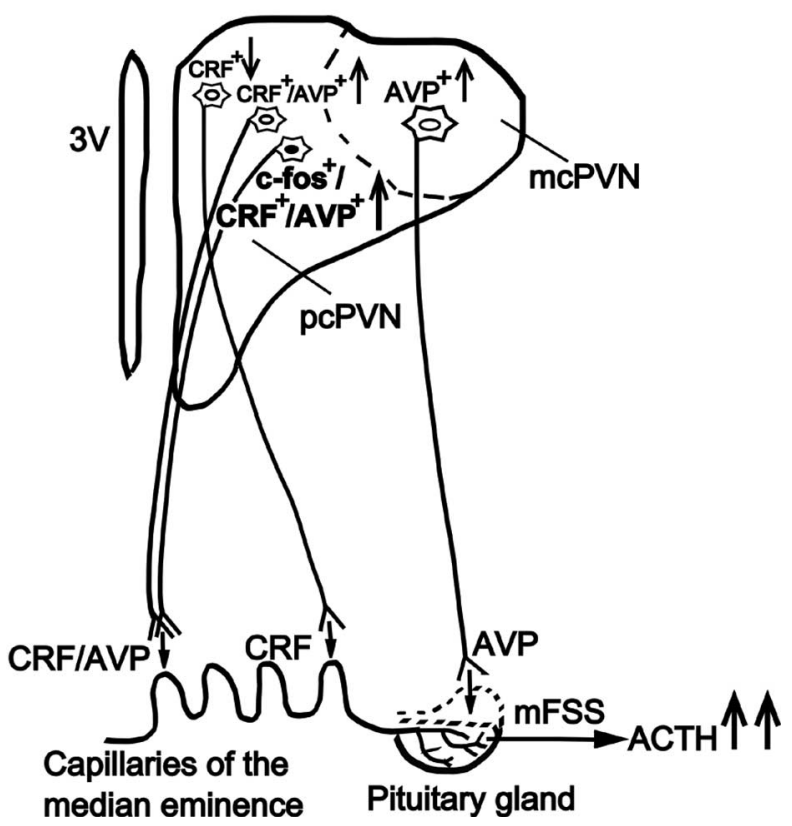

Figure 8. Schematic of PVN neuroadaptive changes and HPA axis responses during nicotine SA. $\boldsymbol{A}$, Effects of nicotine SA per se on ACTH secretion during acquisition of the behavior on days 1 and 3 (NC, no change). $\boldsymbol{B}$, Effects of chronic nicotine SA on the PVN expression of CRF and AVP mRNAs in single- and double-positive neurons within pcPVN and mcPVN and on the ACTH response to $\mathrm{mFSS}$. Chronic nicotine $S A$ increased $(\uparrow)$ the number of $\mathrm{CRF}^{+} / \mathrm{AVP}^{+}$neurons, the number of such neurons activated by $\mathrm{mFSS}\left(\mathrm{c}-\mathrm{FoS}^{+} / \mathrm{CRF}^{+} / \mathrm{AVP}^{+}\right)$, and the ACTH response to $\mathrm{mFSS}$. fold to threefold increase in AVP mRNA expression within pcPVN found in our studies may facilitate the chronic adaptation of the HPA axis to the reduction in CRF, thereby maintaining stable basal ACTH and corticosterone levels and enhancing the responsiveness to selective stressors during chronic nicotine SA, as reported recently (Chen et al., 2007a). Additionally, enhanced AVP expression may be involved in maintaining stable HPA responsiveness to certain stressors that might otherwise be associated with blunted HPA responses attributable to the reduction in CRF expression.

Magnocellular AVP mRNA expression also was increased by chronic nicotine SA, consistent with reports showing that mcPVN AVP mRNA was increased by the chronic stressors cold, isolation, and intermittent walking stress (Angulo et al., 1991; Nakase et al., 1998). Serum osmolarity, another potential mechanism for enhanced mcPVN AVP mRNA expression, remained constant throughout chronic nicotine SA. Although it is well established that mcPVN AVP is transported to the neural pituitary lobe and secreted into peripheral circulation, electrophysiological and morphological evidence suggests that AVP from mcPVN neurons may also gain access to pituitary portal circulation (Antoni, 1993). From this perspective, increased mcPVN AVP mRNA expression, and the implied increase in AVP within the pituitary portal circulation, might also contribute to adaptation of the HPA during chronic nicotine SA.

Increased AVP expression in pcPVN CRF ${ }^{+}$neurons contributes to the sensitization of pituitary corticotropes to heterotypic stressors (Aguilera and Rabadan-Diehl, 2000). Because chronic nicotine SA enhanced the HPA responses to a novel stressor (mFSS) (Chen et al., 2007a), we studied c-Fos activation in PVN neurons. Consistent with a previous report (Pagliusi et al., 1996), we observed that very few PVN neurons of either nicotine or saline SA rats expressed c-Fos (supplemental Fig. 1, available at www.jneurosci.org as supplemental material), whereas mFSS significantly increased c-Fos expression in both $\mathrm{CRF}^{+}$and $\mathrm{AVP}^{+}$ neurons (Figs. 6, 7). Chronic nicotine SA did not affect the number of $\mathrm{c}-\mathrm{Fos}^{+} / \mathrm{CRF}^{+}$neurons activated by mFSS, and, because the total $\mathrm{CRF}^{+}$number was reduced $65 \%$, there was a large increase in the percentage of $\mathrm{CRF}^{+}$neurons activated by mFSS compared with saline SA ( 85 vs $60 \%$ ). However, the numbers of $\mathrm{c}-F o s^{+} / \mathrm{AVP}^{+}$and $\mathrm{c}-\mathrm{Fos}^{+} / \mathrm{CRF}^{+} / \mathrm{AVP}^{+}$neurons activated by $\mathrm{mFSS}$ in pcPVN were increased significantly after chronic nicotine SA. Therefore, chronic nicotine SA not only increased expression of $\mathrm{AVP}^{+}$in pcPVN, it also enhanced the activation of these neurons (i.e., both $\mathrm{AVP}^{+}$and $\mathrm{CRF}^{+} / \mathrm{AVP}^{+}$) by a novel stressor. This is likely to result in enhanced AVP secretion into pituitary portal circulation and, consequently, in potentiation of CRF-induced ACTH release, which would be an important factor in the cross-sensitization to mFSS after chronic nicotine SA.

Acute nicotine activated $\mathrm{CRF}^{+}$neurons in adult rat PVN (Valentine et al., 1996; Loughlin et al., 2006) but did not change their number (Loughlin et al., 2006). Additionally, nicotine increased CRF mRNA in an immortalized amygdala cell line (Kasckow et al., 1999), hypothalamic CRF-like immunoreactivity was increased in adult rats exposed to nicotine during adolescence (Slawecki et al., 2005), and hypothalamic provasopressin mRNA increased on day 1-4 of daily nicotine injection (Hollt and Horn, 1992). However, we consistently observed a reduction in CRF mRNA and an increase in AVP mRNA during chronic nicotine SA. Therefore, the effects of nicotine on CRF and AVP mRNA expression appear to depend on the specific parameters of the experimental model. Similarly, the state of the animal (i.e., stable nicotine SA vs withdrawal) appears to modify HPA axis respon- 
siveness. For example, Semba et al. (2004) showed that nicotine withdrawal reduced the sensitivity of the HPA axis to restraint stress, whereas we reported that HPA responsiveness to mild but not strong footshock was enhanced during chronic nicotine SA (Chen et al., 2007a).

We proposed previously that nicotine SA functions as a chronic stressor (Chen et al., 2007a) based on the observation that ACTH and corticosterone responses induced by selfadministered nicotine desensitized over time, and chronic nicotine SA cross-sensitized the stress response to a novel stressor. The current results demonstrate that chronic nicotine SA modulated CRF and AVP mRNA expression and enhanced the response of $\mathrm{CRF}^{+} / \mathrm{AVP}^{+}$neurons to a heterotypic stressor, providing additional support for this concept. The cross-sensitization of HPA responses to a novel stressor during chronic nicotine SA indicates that smoking may increase stress responsiveness in cigarette smokers, similar to reports showing that smokers report higher perceived levels of stress (Croghan et al., 2006; Finkelstein et al., 2006). Because stressors can induce craving in paradigms modeling reinstatement of drug seeking, it is conceivable that nicotine enhances the efficacy of stressors to induce craving for cigarettes.

In conclusion, chronic nicotine SA significantly decreased CRF and increased AVP mRNA expression, increasing their colocalization in $\mathrm{pcPVN}$; this population of $\mathrm{CRF}^{+} / \mathrm{AVP}^{+}$neurons accounted for a greater number of the CRF neurons activated by $\mathrm{mFSS}$ in pcPVN of nicotine SA rats. These phenotypic changes in $\mathrm{pcPVN}$ neurons may be involved in desensitization of HPA responses to nicotine stimulation and in cross-sensitization of HPA responses to a specific novel heterotypic stressor during chronic nicotine SA. Thus, modulation of the phenotype of pcPVN neurons by chronic nicotine SA is pivotal in augmenting the HPA response to specific stressors.

\section{References}

Aguilera G (1994) Regulation of pituitary ACTH secretion during chronic stress. Front Neuroendocrinol 15:321-350.

Aguilera G, Rabadan-Diehl C (2000) Vasopressinergic regulation of the hypothalamic-pituitary-adrenal axis: implications for stress adaptation. Regul Pept 96:23-29.

Angulo JA, Ledoux M, McEwen BS (1991) Genomic effects of cold and isolation stress on magnocellular vasopressin mRNA-containing cells in the hypothalamus of the rat. J Neurochem 56:2033-2038.

Antoni FA (1993) Vasopressinergic control of pituitary adrenocorticotropin secretion comes of age. Front Neuroendocrinol 14:76-122.

Balfour DJ, Khullar AK, Longden A (1975) Effects of nicotine on plasma corticosterone and brain amines in stressed and unstressed rats. Pharmacol Biochem Behav 3:179-184.

Bartanusz V, Jezova D, Bertini LT, Tilders FJ, Aubry JM, Kiss JZ (1993) Stress-induced increase in vasopressin and corticotropin-releasing factor expression in hypophysiotrophic paraventricular neurons. Endocrinology 132:895-902.

Cam GR, Bassett JR (1983) The effect of acute nicotine administration on plasma levels of the thyroid hormones and corticosterone in the rat. Pharmacol Biochem Behav 19:559-561.

Chen H, Hansen MJ, Jones JE, Vlahos R, Bozinovski S, Anderson GP, Morris MJ (2006) Cigarette smoke exposure reprograms the hypothalamic neuropeptide Y axis to promote weight loss. Am J Respir Crit Care Med 173:1248-1254.

Chen H, Fu Y, Sharp BM (2007a) Chronic nicotine self-administration augments hypothalamic-pituitary-adrenal responses to mild acute stress. Neuropsychopharmacology 33:721-730.

Chen H, Hansen MJ, Jones JE, Vlahos R, Bozinovski S, Anderson GP, Morris MJ (2007b) Regulation of hypothalamic NPY by diet and smoking. Peptides 28:384-389.

Conte-Devolx B, Oliver C, Giraud P, Gillioz P, Castanas E, Lissitzky JC,
Boudouresque F, Millet Y (1981) Effect of nicotine on in vivo secretion of melanocorticotropic hormones in the rat. Life Sci 28:1067-1073.

Croghan IT, Bronars C, Patten CA, Schroeder DR, Nirelli LM, Thomas JL, Clark MM, Vickers KS, Foraker R, Lane K, Houlihan D, Offord KP, Hurt $\mathrm{RD}$ (2006) Is smoking related to body image satisfaction, stress, and self-esteem in young adults? Am J Health Behav 30:322-333.

de Goeij DC, Kvetnansky R, Whitnall MH, Jezova D, Berkenbosch F, Tilders FJ (1991) Repeated stress-induced activation of corticotropin-releasing factor neurons enhances vasopressin stores and colocalization with corticotropin-releasing factor in the median eminence of rats. Neuroendocrinology 53:150-159.

de Goeij DC, Jezova D, Tilders FJ (1992) Repeated stress enhances vasopressin synthesis in corticotropin releasing factor neurons in the paraventricular nucleus. Brain Res 577:165-168.

Finkelstein DM, Kubzansky LD, Goodman E (2006) Social status, stress, and adolescent smoking. J Adolesc Health 39:678-685.

Fu Y, Matta SG, Brower VG, Sharp BM (2001) Norepinephrine secretion in the hypothalamic paraventricular nucleus of rats during unlimited access to self-administered nicotine: an in vivo microdialysis study. J Neurosci 21:8979-8989.

Fu Y, Matta SG, Kane VB, Sharp BM (2003) Norepinephrine release in amygdala of rats during chronic nicotine self-administration: an in vivo microdialysis study. Neuropharmacology 45:514-523.

Gillies GE, Linton EA, Lowry PJ (1982) Corticotropin releasing activity of the new CRF is potentiated several times by vasopressin. Nature 299:355-357.

Grinevich V, Ma XM, Herman JP, Jezova D, Akmayev I, Aguilera G (2001) Effect of repeated lipopolysaccharide administration on tissue cytokine expression and hypothalamic-pituitary-adrenal axis activity in rats. J Neuroendocrinol 13:711-723.

Harbuz MS, Lightman SL (1992) Stress and the hypothalamo-pituitaryadrenal axis: acute, chronic and immunological activation. J Endocrinol 134:327-339.

Hauger RL, Millan MA, Lorang M, Harwood JP, Aguilera G (1988) Corticotropin-releasing factor receptors and pituitary adrenal responses during immobilization stress. Endocrinology 123:396-405.

Hollt V, Horn G (1992) Effect of nicotine on mRNA levels encoding opioid peptides, vasopressin and alpha 3 nicotinic receptor subunit in the rat. Clin Investig 70:224-231.

Johnstone LE, Srisawat R, Kumarnsit E, Leng G (2005) Hypothalamic expression of NPY mRNA, vasopressin mRNA and CRF mRNA in response to food restriction and central administration of the orexigenic peptide GHRP-6. Stress 8:59-67.

Kasckow JW, Regmi A, Sheriff S, Mulchahey J, Geracioti Jr TD (1999) Regulation of corticotropin-releasing factor messenger RNA by nicotine in an immortalized amygdalar cell line. Life Sci 65:2709-2714.

Koval JJ, Pederson LL, Chan SS (2004) Psychosocial variables in a cohort of students in grades 8 and 11: a comparison of current and never smokers. Prev Med 39:1017-1025.

Lolait SJ, Stewart LQ, Jessop DS, Young III WS, O'Carroll AM (2007) The hypothalamic-pituitary-adrenal axis response to stress in mice lacking functional vasopressin V1b receptors. Endocrinology 148:849-856.

Loughlin SE, Islas MI, Cheng MY, Lee AG, Villegier AS, Leslie FM (2006) Nicotine modulation of stress-related peptide neurons. J Comp Neurol 497:575-588.

Ma XM, Lightman SL (1998) The arginine vasopressin and corticotrophinreleasing hormone gene transcription responses to varied frequencies of repeated stress in rats. J Physiol (Lond) 510:605-614.

Ma XM, Lightman SL, Aguilera G (1999) Vasopressin and corticotropinreleasing hormone gene responses to novel stress in rats adapted to repeated restraint. Endocrinology 140:3623-3632.

Matta SG, Beyer HS, McAllen KM, Sharp BM (1987) Nicotine elevates rat plasma ACTH by a central mechanism. J Pharmacol Exp Ther 243:217-226.

Matta SG, Fu Y, Valentine JD, Sharp BM (1998) Response of the hypothalamo-pituitary-adrenal axis to nicotine. Psychoneuroendocrinology 23:103-113.

McNair E, Bryson R (1983) Effects of nicotine on weight change and food consumption in rats. Pharmacol Biochem Behav 18:341-344.

Nakase S, Kitayama I, Soya H, Hamanaka K, Nomura J (1998) Increased expression of magnocellular arginine vasopressin mRNA in paraventricular nucleus of stress-induced depression-model rats. Life Sci 63:23-31. 
Pagliusi SR, Tessari M, DeVevey S, Chiamulera C, Pich EM (1996) The reinforcing properties of nicotine are associated with a specific patterning of c-fos expression in the rat brain. Eur J Neurosci 8:2247-2256.

Parrott AC (1995) Stress modulation over the day in cigarette smokers. Addiction 90:233-244

Paxinos G, Watson C (1986) The rat brain in stereotaxic coorinates, Ed 2. New York: Academic.

Potter E, Sutton S, Donaldson C, Chen R, Perrin M, Lewis K, Sawchenko PE, Vale W (1994) Distribution of corticotropin-releasing factor receptor mRNA expression in the rat brain and pituitary. Proc Natl Acad Sci USA 91:8777-8781.

Rho JH, Swanson LW (1989) A morphometric analysis of functionally defined subpopulations of neurons in the paraventricular nucleus of the rat with observations on the effects of colchicine. J Neurosci 9:1375-1388.

Rivier C, Vale W (1983) Interaction of corticotropin-releasing factor and arginine vasopressin on adrenocorticotropin secretion in vivo. Endocrinology 113:939-942.

Schepis TS, Rao U (2005) Epidemiology and etiology of adolescent smoking. Curr Opin Pediatr 17:607-612.

Semba J, Wakuta M, Maeda J, Suhara T (2004) Nicotine withdrawal induces subsensitivity of hypothalamic-pituitary-adrenal axis to stress in rats: implications for precipitation of depression during smoking cessation. Psychoneuroendocrinology 29:215-226.

Slawecki CJ, Thorsell AK, El Khoury A, Mathe AA, Ehlers CL (2005) In- creased CRF-like and NPY-like immunoreactivity in adult rats exposed to nicotine during adolescence: relation to anxiety-like and depressive-like behavior. Neuropeptides 39:369-377.

Valentine JD, Matta SG, Sharp BM (1996) Nicotine-induced cFos expression in the hypothalamic paraventricular nucleus is dependent on brainstem effects: correlations with cFos in catecholaminergic and noncatecholaminergic neurons in the nucleus tractus solitarius. Endocrinology 137:622-630.

Valentine JD, Hokanson JS, Matta SG, Sharp BM (1997) Selfadministration in rats allowed unlimited access to nicotine. Psychopharmacology (Berl) 133:300-304.

Zhao R, Chen H, Sharp BM (2007) Nicotine-induced norepinephrine release in hypothalamic paraventricular nucleus and amygdala is mediated by $N$-methyl-D-aspartate receptors and nitric oxide in the nucleus tractus solitarius. J Pharmacol Exp Ther 320:837-844.

Zhou Y, Spangler R, LaForge KS, Maggos CE, Ho A, Kreek MJ (1996) Corticotropin-releasing factor and type 1 corticotropin-releasing factor receptor messenger RNAs in rat brain and pituitary during "binge"pattern cocaine administration and chronic withdrawal. J Pharmacol Exp Ther 279:351-358.

Zhou Y, Bendor JT, Yuferov V, Schlussman SD, Ho A, Kreek MJ (2005) Amygdalar vasopressin mRNA increases in acute cocaine withdrawal: evidence for opioid receptor modulation. Neuroscience 134:1391-1397. 